\title{
Modern Contraceptive and Dual Method Use among HIV-Infected Women in Lusaka, Zambia
}

\author{
Carla J. Chibwesha, ${ }^{1,2}$ Michelle S. Li, ${ }^{1,2}$ Christine K. Matoba, ${ }^{2}$ Reuben K. Mbewe, ${ }^{3}$ \\ Benjamin H. Chi, ${ }^{1,2}$ Jeffrey S. A. Stringer, ${ }^{1,2}$ and Elizabeth M. Stringer ${ }^{1,2}$ \\ ${ }^{1}$ University of Alabama School of Medicine, 1530 Third Avenue South, CRWH-379, Birmingham, AL 35294, USA \\ ${ }^{2}$ Centre for Infectious Disease Research in Zambia, P.O. Box 34681, Lusaka 10101, Zambia \\ ${ }^{3}$ Zambian Ministry of Health, Ndeke House, Lusaka 10101, Zambia
}

Correspondence should be addressed to Carla J. Chibwesha, carla.chibwesha@cidrz.org

Received 15 April 2011; Revised 14 June 2011; Accepted 6 July 2011

Academic Editor: Maura K. Whiteman

Copyright (๑) 2011 Carla J. Chibwesha et al. This is an open access article distributed under the Creative Commons Attribution License, which permits unrestricted use, distribution, and reproduction in any medium, provided the original work is properly cited.

\begin{abstract}
HIV-infected women in sub-Saharan Africa are at substantial risk of unintended pregnancy and sexually transmitted infections (STIs). Linkages between HIV and reproductive health services are advocated. We describe implementation of a reproductive health counseling intervention in 16 HIV clinics in Lusaka, Zambia. Between November 2009 and November 2010, 18,407 women on antiretroviral treatment (ART) were counseled. The median age was 34.6 years (interquartile range (IQR): 29.9-39.7), and $60.1 \%$ of women were married. The median $\mathrm{CD}^{+}$cell count was 394 cells/uL (IQR: $256-558$ ). Of the women counseled, 10,904 (59.2\%) reported current modern contraceptive use. Among contraceptive users, only $17.7 \%$ reported dual method use. After counseling, 737 of 7,503 women not previously using modern contraception desired family planning referrals, and $61.6 \%$ of these women successfully accessed services within 90 days. Unmet contraceptive need remains high among HIV-infected women. Additional efforts are needed to promote reproductive health, particularly dual method use.
\end{abstract}

\section{Introduction}

The Millennium Development Goals, adopted in New York in 2000, promote universal education and gender equality, maternal and child health, and prevention and treatment for HIV/AIDS [1]. Provision of comprehensive reproductive health care is central to attaining these goals [2]. Worldwide, as many as one-third of the 357,000 annual maternal deaths are attributable to unintended pregnancies; the majority of these mortalities occur in low- and middle-income countries [3-6]. Enhanced access to family planning services in sub-Saharan Africa would result in marked reductions in unintended pregnancies and unsafe abortions and a projected $69 \%$ decrease in maternal deaths and a $57 \%$ decrease in newborn deaths [4]. In addition to substantial risks of dying from pregnancy complications [7], women in a sub-Saharan Africa are also at increased risk of HIV and other sexually transmitted infections $[8,9]$. Providing safe, effective contraception to HIV-infected women who desire it has also been identified by the World Health Organization as a primary strategy for prevention of pediatric infections [10].

In Zambia, as in many other sub-Saharan African countries, HIV-infected women of childbearing age represent a vulnerable population. The burden of STIs is high among HIV-infected pregnant women in this setting [11]. Additionally, only $20-40 \%$ of HIV-infected Zambian couples, whether serodiscordant or concordant, report use of a modern contraceptive method other than condoms [12, 13]. Public health programs that emphasize dual family planning methods-highly effective modern contraception coupled with condom use-will ensure protection from both unintended pregnancy, and STIs, and should form the cornerstone of reproductive health care. In HIV care and treatment programs, medication adherence counseling provides a unique window of opportunity to address preventive health recommendations, including family planning and STI prevention. In this paper, we describe implementation of 
an integrated, reproductive health peer counselor program in 16 public-sector HIV clinics in Lusaka, Zambia. We also report (1) baseline modern contraceptive and dual method use among HIV-infected women receiving antiretroviral treatment, (2) uptake of modern contraceptive and dual methods following peer counseling, and (3) predictors of modern contraceptive and dual method use among HIVinfected women.

\section{Materials and Methods}

Lusaka is home to almost 2 million people [14]. The HIV prevalence rate among pregnant women is $21 \%$ [15], and the majority of women infected with HIV are of reproductive age. The Ministry of Health's ART program was established in 2004 and covers the entire city. Over 100,000 HIVinfected individuals in Lusaka are now receiving care in this system. Due to the large volumes of patients and human resource shortages [16], peer educators conduct most ART counseling sessions. Prior to our intervention, neither routine family planning nor dual method counseling was provided during peer counseling sessions. To expand the scope and effectiveness of these counseling visits, we designed and implemented a reproductive health peer counselor program integrated within 16 primary care HIV clinics in Lusaka.

We trained 109 peer counselors to deliver a standardized counseling message, emphasizing dual methods. The counseling intervention was implemented within the context of routine clinical care. With the aid of a printed counseling tool, peer educators delivered a comprehensive reproductive health message, including information on the range of barrier methods, hormonal and intrauterine contraception, and permanent sterilization. Women who desired access to reproductive health services were referred to a separate, on-site family planning department. In order to support public-sector reproductive health service provision, we also trained 42 family planning nurses. Training was based on the national family planning curriculum and nurses who successfully completed both a classroom-based course and a mentored, clinical practicum-received certification. Public-sector family planning clinics provided condoms, oral contraceptive pills (OCPs), depot medroxyprogesterone acetate (DMPA), Jadelle levonorgestrel implants, and copper intrauterine devices (IUDs). Women who wished to undergo permanent sterilization were referred to a center with surgical facilities, such as the University Teaching Hospital.

Our analysis cohort included HIV-infected women aged 16-50 years and on ART at one of the 16 intervention clinics. To be eligible, a woman had to have at least one reproductive health counseling visit documented in her medical record between November 2009 and November 2010. We report baseline sociodemographic data, $\mathrm{CD} 4^{+}$cell count (cells/uL), hemoglobin level $(\mathrm{g} / \mathrm{dL})$, and history of tuberculosis. These data were ascertained through review of women's electronic medical and laboratory records. Laboratory results were assessed within 90 days of the woman's counseling visit. We also report use of modern contraception, including dual method use. Peer counselors collected data relating to reproductive health counseling and contraceptive use on a clinical form developed for the project. We considered condoms, OCPs, DMPA, Jadelle, IUDs, and sterilization as modern contraception. Dual method use was defined as use of condoms to prevent STIs coupled with use of a short- or long-term reversible contraceptive or sterilization. Contraceptive use data were self-reported.

Univariate and multivariate regression analyses were used to identify sociodemographic and other predictors independently associated with modern contraceptive and dual method use, as well as with access to family planning services within 90 days of a counseling visit. Crude odds ratios (ORs) and 95\% confidence intervals (CIs) were computed using logistic regression models. Adjusted odds ratios (AORs) and their corresponding 95\% CIs were generated using generalized estimating equations to account for clustering at the site level. All statistical analyses were performed using SAS version 9.1.3 (SAS Institute Inc, Cary, North Carolina). Ethical approval for this study was obtained from the University of Zambia Biomedical Research Ethics Committee (Lusaka, Zambia) and the University of Alabama at Birmingham Institutional Review Board (Birmingham, AL, USA).

\section{Results}

Between November 2009 and November 2010, 32,998 women had a least one clinic visit at a site where the counseling program had been implemented. Project staffing levels had been calculated on an estimated 20,000 clinical visits during the one-year implementation period. Therefore, for logistic reasons, not all women accessing HIV treatment services at participating clinics received reproductive health counseling. Over the study period, documented reproductive health counseling visits were available for 18,407 (55.8\%) HIV-infected women. Baseline characteristics of women who completed at least one counseling visit compared with those who did not receive reproductive health counseling are detailed in Table 1. With the exception of median $\mathrm{CD} 4^{+}$cell count and median treatment duration, differences between women who received the counseling intervention and those who did not were not clinically significant.

3.1. Cohort Description. Our analysis cohort included 18,407 HIV-infected women. At enrolment, the median age was 34.6 years (IQR: 29.9-39.7 years), $60.1 \%$ of women were married, $87.4 \%$ had one or more living children, and $39.2 \%$ had completed some secondary education. $63.0 \%$ of women reported their monthly income as $\geq 200,000$ Zambian kwacha (approximately $\$ 45$ ). The median $\mathrm{CD} 4^{+}$ cell count was 394 cells/uL (IQR: 256-558 cells/uL), and the median hemoglobin level was $12.4 \mathrm{~g} / \mathrm{dL}$ (IQR: $11.3-$ $13.4 \mathrm{~g} / \mathrm{dL}$ ). The median time on ART was 709 days (IQR: 262-1,302 days). 3,441 (18.7\%) women reported a history of or current active tuberculosis infection. Less than half $(42.5 \%)$ of the women in our cohort had disclosed their serostatus to a partner. Furthermore, most women (77.1\%) did not know or did not provide information about their partner's HIV status at the time of enrollment into care. 
TABLE 1: Baseline characteristics of HIV-infected women aged 16-50 years receiving antiretroviral treatment at 16 public-sector clinics in Lusaka, Zambia (November 2009-November 2010).

\begin{tabular}{|c|c|c|c|c|c|}
\hline & Women with & nted RH counseling & Women with $\mathrm{n}$ & nented RH counseling visit & $P$-value \\
\hline & $N$ & Value & $N$ & Value & \\
\hline $\begin{array}{l}\text { Age at 1st visit (years), median } \\
\text { (IQR) }\end{array}$ & 18,407 & $34.6(29.9-39.7)$ & 14,591 & $34.7(29.5-40.9)$ & $<0.001^{++}$ \\
\hline $16-24$ & 1,246 & $6.8 \%$ & 1,263 & $8.7 \%$ & $<0.001^{* *}$ \\
\hline $25-34$ & 8,362 & $45.4 \%$ & 6,232 & $42.7 \%$ & \\
\hline$\geq 35$ & 8,799 & $47.8 \%$ & 7,096 & $48.6 \%$ & \\
\hline Marital status & 14,180 & & 11,135 & & \\
\hline Married & 8,523 & $60.1 \%$ & 6,570 & $59.0 \%$ & $0.076^{* *}$ \\
\hline Single/divorced/widowed & 5,657 & $39.9 \%$ & 4,565 & $41.0 \%$ & \\
\hline Education & 16,910 & & 13,441 & & \\
\hline None & 4,677 & $27.7 \%$ & 3,785 & $28.2 \%$ & $0.016^{* *}$ \\
\hline Primary & 5,603 & $33.1 \%$ & 4,246 & $31.6 \%$ & \\
\hline Secondary or more & 6,630 & $39.2 \%$ & 5,410 & $40.2 \%$ & \\
\hline $\begin{array}{l}\text { Number of living children, } \\
\text { median (IQR) }\end{array}$ & 14,385 & $2(1-3)$ & 11,373 & $2(1-3)$ & $0.061^{++}$ \\
\hline 0 & 1,806 & $12.6 \%$ & 1,505 & $13.2 \%$ & $0.106^{* *}$ \\
\hline$\geq 1$ & 12,579 & $87.4 \%$ & 9,868 & $86.8 \%$ & \\
\hline Monthly income & 12,243 & & 9,400 & & \\
\hline$<$ ZMK 200,000 & 4,532 & $37.0 \%$ & 3,864 & $41.1 \%$ & $<0.001^{* *}$ \\
\hline$\geq \mathrm{ZMK} 200,000$ & 7,711 & $63.0 \%$ & 5,536 & $58.9 \%$ & \\
\hline $\begin{array}{l}\mathrm{CD}^{+} \text {cell count (cells/uL), } \\
\text { median }(\mathrm{IQR})\end{array}$ & 18,231 & $394(256-558)$ & 14,419 & $357(228-522)$ & $<0.001^{++}$ \\
\hline$<250$ & 4,361 & $23.9 \%$ & 4,187 & $29.0 \%$ & $<0.001^{* *}$ \\
\hline $250-350$ & 3,338 & $18.3 \%$ & 2,819 & $19.6 \%$ & \\
\hline$\geq 351$ & 10,532 & $57.8 \%$ & 7,413 & $51.4 \%$ & \\
\hline $\begin{array}{l}\text { Hemoglobin }(\mathrm{g} / \mathrm{dL}) \text {, median } \\
\text { (IQR) }\end{array}$ & 18,175 & $12.4(11.3-13.4)$ & 14,248 & $12.2(11.0-13.1)$ & $<0.001^{++}$ \\
\hline$\leq 8.0$ & 363 & $2.0 \%$ & 482 & $3.4 \%$ & $<0.001^{* *}$ \\
\hline $8.1-9.9$ & 1,365 & $7.5 \%$ & 1,276 & $9.0 \%$ & \\
\hline$\geq 10.0$ & 16,447 & $90.5 \%$ & 12,490 & $87.7 \%$ & \\
\hline History or current tuberculosis & 18,407 & & 14,591 & & \\
\hline Yes & 3,441 & $18.7 \%$ & 2,771 & $19.0 \%$ & $0.493^{* *}$ \\
\hline No & 14,966 & $81.3 \%$ & 11,820 & $81.0 \%$ & \\
\hline $\begin{array}{l}\text { Time on ART (days), median } \\
\text { (IQR) }\end{array}$ & 18,407 & $709(262-1,302)$ & 14,591 & $659(245-1,180)$ & $<0.001^{++}$ \\
\hline HIV status disclosure to partner & 18,407 & & 14,591 & & \\
\hline Yes & 7,823 & $42.5 \%$ & 6,026 & $41.3 \%$ & $0.028^{* *}$ \\
\hline Unknown & 10,584 & $57.5 \%$ & 8,565 & $58.7 \%$ & \\
\hline Partner's HIV status & 18,407 & & 14,591 & & \\
\hline Negative & 621 & $3.4 \%$ & 456 & $3.1 \%$ & $<0.001^{* *}$ \\
\hline Positive & 3,598 & $19.5 \%$ & 2,599 & $17.8 \%$ & \\
\hline Unknown & 5,332 & $29.0 \%$ & 4,362 & $29.9 \%$ & \\
\hline Missing & 8,856 & $48.1 \%$ & 7,174 & $49.2 \%$ & \\
\hline
\end{tabular}

RH: reproductive health; IQR: interquartile range; ZMK: Zambian Kwacha; ART: antiretroviral therapy.

** Person's chi-square test; ${ }^{++}$Wilcoxon rank sum test. 


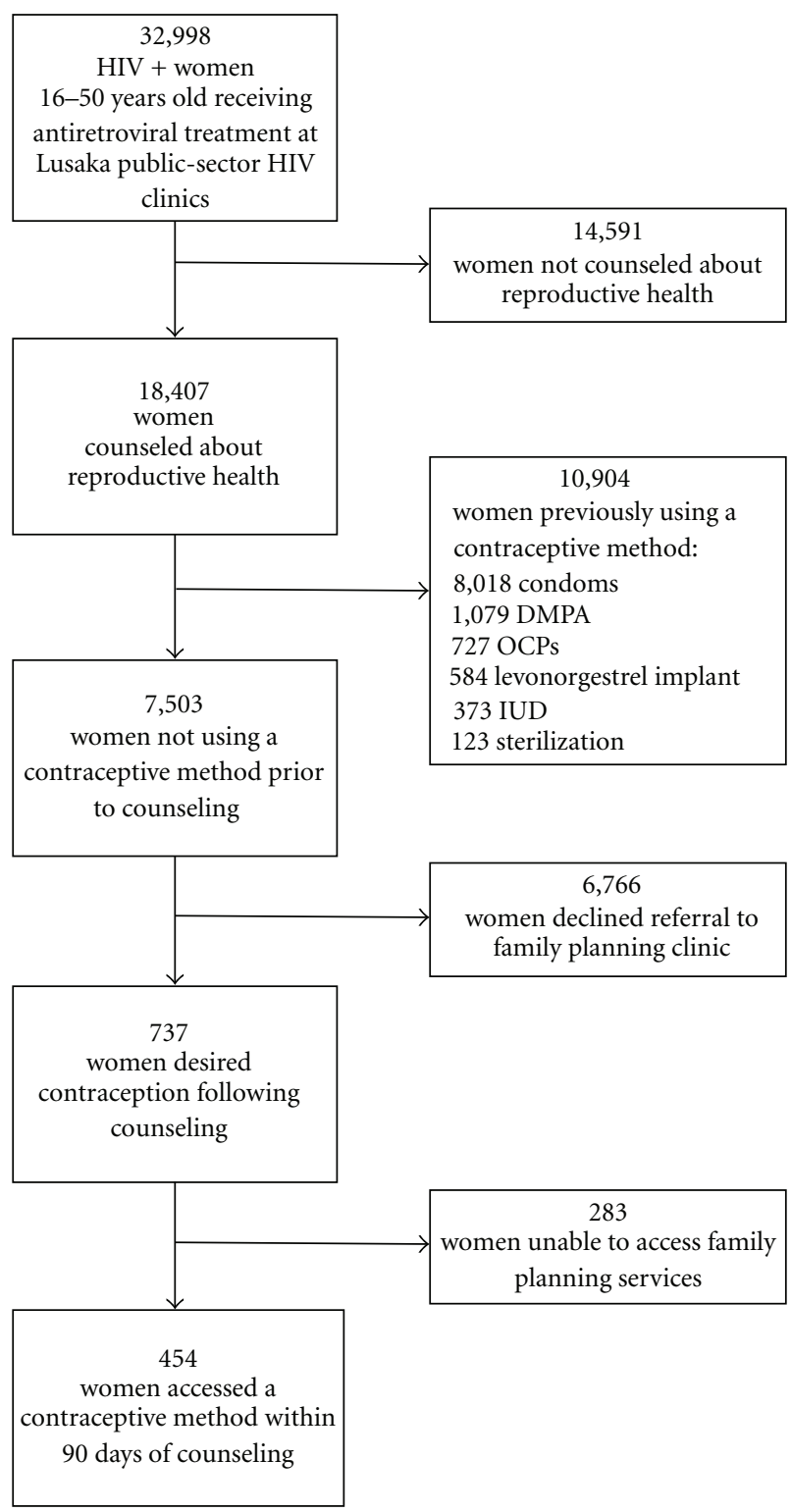

Figure 1: Description of the study cohort.

3.2. Baseline Modern Contraceptive and Dual Method Use. Of the 18,407 women included in the analysis, $10,904(59.2 \%)$ reported current use of a modern contraceptive method: $73.5 \%$ of women reported condom use, 9.9\% DMPA use, 6.7\% OCP use, 5.4\% levonorgestrel implant use, $3.4 \%$ IUD use, and $1.1 \%$ had undergone permanent sterilization (Figure 1). Among the 10,904 women who reported use of a modern contraceptive method at their counseling visit, $1,927(17.7 \%)$ stated that they used dual methods for both pregnancy and STI prevention.

3.3. Impact of the Counseling Intervention and Unmet Contraceptive Need. Of the 7,503 (40.8\%) women in our cohort not using modern contraception, 737 (9.8\%) women desired contraception after counseling and 71 stated an intention to use dual methods. 454 of 737 (61.6\%) women who desired contraception successfully accessed family planning services within 90 days of their counseling visit. Our data, therefore, indicate that nearly $40 \%$ of women who desired reproductive health services were unable to access public-sector services (i.e., free from point-of-service user fees). This represents substantial unmet contraceptive need within Lusaka's public health system.

In univariate analysis, age was the only factor associated with successful access to contraceptive services within 90 days of a counseling visit. In multivariate analysis, women 25-34 years (AOR: 0.53; 95\% CI: 0.30-0.92) or $\geq 35$ years (AOR: 0.49; 95\% CI: 0.25-1.00) had lower odds of accessing contraceptive services than women 16-24 years. Women who reported a higher monthly income also had lower odds of accessing contraceptive services than women who were less wealthy (AOR: 0.68; 95\% CI: 0.47-0.98). By contrast, multiparae were more likely to access reproductive health services within 90 days than women with no living children (AOR: 1.83; 95\% CI: 1.17-2.88).

3.4. Predictors of Modern Contraceptive Use. In univariate analysis, women were less likely to report modern contraceptive use if $\geq 35$ years, single, divorced, or widowed, with undisclosed HIV status, and if their partner's HIV status was unknown (Table 2). Conversely, women 25-34 years with one or more living children, those reporting higher monthly incomes, those with $\mathrm{CD}^{+}$cell counts $\geq 250$ cells $/ \mathrm{uL}$ or hemoglobin levels $\geq 8.1 \mathrm{~g} / \mathrm{dL}$, and those without a history of tuberculosis infection had increased odds of contraceptive use. In multivariate analysis, age, marital status, HIV status disclosure, parity, $\mathrm{CD}^{+}$cell count, and hemoglobin level remained associated with modern contraceptive use. Women $\geq 35$ years old (AOR: 0.63; 95\% CI: 0.52-0.77), single, divorced, or widowed women (AOR: 0.30; 95\% CI: $0.27-$ 0.34), and those for whom HIV status disclosure was unknown (AOR: 0.75; 95\% CI: 0.64-0.87) had lower odds of using modern contraception. The odds of using modern contraception were higher among women with one or more living children (AOR: 1.47; 95\% CI: 1.21-1.78), and among healthier women with $\mathrm{CD} 4^{+}$cell counts of $250-350$ cells/uL (AOR: 1.18 ; 95\% CI: $1.05-1.33$ ) or $\geq 351$ cells/uL (AOR: 1.23 ; 95\% CI: 1.10-1.38) than among nullipara and those with low $\mathrm{CD}^{+}$cell counts. A similar association was observed for hemoglobin levels 8.1-9.9 g/dL (AOR: 1.56; 95\% CI: 1.241.97 ) and $\geq 10.0 \mathrm{~g} / \mathrm{dL}$ (AOR: 2.16; 95\% CI: 1.72-2.71).

3.5. Predictors of Dual Method Use. In univariate analysis, women who were $\geq 35$ years, single, divorced, or widowed and those who did not provide information regarding HIV status disclosure had a lower odds of dual method use (Table 3). Higher odds of dual method use were observed among women with one or more living children, those with $\mathrm{CD}^{+}$cell counts $\geq 351$ cells/uL, and those who did not report a history of tuberculosis. In multivariate analysis, women $\geq 35$ years (AOR: 0.51; 95\% CI: 0.41-0.63) were less likely to report dual method use than those 16-24 years. Women who were single, divorced, or widowed were also less likely to use dual methods than married women (AOR: 
TABle 2: Predictors of modern contraceptive use among HIV-infected women aged 16-50 years receiving antiretroviral treatment at 16 public-sector clinics in Lusaka, Zambia (November 2009-November 2010).

\begin{tabular}{|c|c|c|c|c|}
\hline & $\begin{array}{l}\text { Women using modern } \\
\text { contraception } \\
\qquad N=11,358\end{array}$ & $\begin{array}{l}\text { Women not using modern } \\
\text { contraception } \\
N=7,049\end{array}$ & Crude OR (95\% CI) & Adjusted OR (95\% CI) \\
\hline \multicolumn{5}{|l|}{ Age at 1st visit (years) } \\
\hline $16-24$ & $736(6.5 \%)$ & $510(7.2 \%)$ & 1.00 & 1.00 \\
\hline $25-34$ & $5,747(50.6 \%)$ & $2,615(37.1 \%)$ & $1.52(1.35-1.72)$ & $1.05(0.87-1.25)$ \\
\hline$\geq 35$ & $4,875(42.9 \%)$ & $3,924(55.7 \%)$ & $0.86(0.76-0.97)$ & $0.63(0.52-0.77)$ \\
\hline \multicolumn{5}{|l|}{ Marital status } \\
\hline Married & $6,503(72.9 \%)$ & $2,020(38.4 \%)$ & 1.00 & 1.00 \\
\hline Single/divorced/widowed & $2,414(27.1 \%)$ & $3,243(61.6 \%)$ & $0.23(0.22-0.25)$ & $0.30(0.27-0.34)$ \\
\hline \multicolumn{5}{|l|}{ Education } \\
\hline None & $2,862(27.4 \%)$ & $1,815(28.2 \%)$ & 1.00 & 1.00 \\
\hline Primary & $3,493(33.4 \%)$ & $2,110(32.7 \%)$ & $1.05(0.97-1.14)$ & $1.13(0.99-1.28)$ \\
\hline Secondary or more & $4,108(39.3 \%)$ & $2,522(39.1 \%)$ & $1.03(0.96-1.12)$ & $1.13(1.00-1.27)$ \\
\hline \multicolumn{5}{|l|}{ Number of living children } \\
\hline 0 & $1,041(11.6 \%)$ & $765(14.2 \%)$ & 1.00 & 1.00 \\
\hline$\geq 1$ & $7,947(88.4 \%)$ & $4,632(85.8 \%)$ & $1.26(1.14-1.39)$ & $1.47(1.21-1.78)$ \\
\hline \multicolumn{5}{|l|}{ Monthly income } \\
\hline$<$ ZMK 200,000 & $2,709(35.9 \%)$ & $1,823(38.8 \%)$ & 1.00 & 1.00 \\
\hline$\geq \mathrm{ZMK} 200,000$ & $4,835(64.1 \%)$ & $2,874(61.2 \%)$ & $1.13(1.05-1.22)$ & $1.03(0.92-1.15)$ \\
\hline \multicolumn{5}{|l|}{$\mathrm{CD}^{+}$cell count $($cells/uL) } \\
\hline$<250$ & $2,473(22.0 \%)$ & $1,887(27.1 \%)$ & 1.00 & 1.00 \\
\hline $250-350$ & $2,042(18.1 \%)$ & $1,295(18.6 \%)$ & $1.20(1.10-1.32)$ & $1.18(1.05-1.33)$ \\
\hline$\geq 351$ & $6,746(59.9 \%)$ & $3,784(54.3 \%)$ & $1.36(1.27-1.46)$ & $1.23(1.10-1.38)$ \\
\hline \multicolumn{5}{|l|}{ Hemoglobin (g/dL) } \\
\hline$\leq 8.0$ & $168(1.5 \%)$ & $195(2.8 \%)$ & 1.00 & 1.00 \\
\hline $8.1-9.9$ & $710(6.3 \%)$ & $655(9.4 \%)$ & $1.26(1.00-1.59)$ & $1.56(1.24-1.97)$ \\
\hline$\geq 10.0$ & $10,355(92.2 \%)$ & $6,090(87.8 \%)$ & $1.97(1.60-2.43)$ & $2.16(1.72-2.71)$ \\
\hline \multicolumn{5}{|l|}{ History or current tuberculosis } \\
\hline Yes & $1,999(17.6 \%)$ & $1,442(20.5 \%)$ & 1.00 & 1.00 \\
\hline No & $9,359(82.4 \%)$ & $5,607(79.5 \%)$ & $1.20(1.12-1.30)$ & $1.06(0.96-1.18)$ \\
\hline \multicolumn{5}{|c|}{ HIV status disclosure to partner } \\
\hline Yes & $5,985(52.7 \%)$ & $1,838(26.1 \%)$ & 1.00 & 1.00 \\
\hline Unknown & $5,373(47.3 \%)$ & $5,211(73.9 \%)$ & $0.32(0.30-0.34)$ & $0.75(0.64-0.87)$ \\
\hline \multicolumn{5}{|l|}{ Partner's HIV status } \\
\hline Negative & $476(4.2 \%)$ & $145(2.1 \%)$ & 1.00 & 1.00 \\
\hline Positive & $2,700(23.8 \%)$ & $898(12.7 \%)$ & $0.92(0.75-1.12)$ & $0.76(0.54-1.06)$ \\
\hline Unknown & $3,357(29.6 \%)$ & $1,975(28.0 \%)$ & $0.52(0.43-0.63)$ & $0.75(0.56-0.99)$ \\
\hline Missing & $4,825(42.5 \%)$ & $4,031(57.2 \%)$ & $0.36(0.30-0.44)$ & $0.66(0.48-0.89)$ \\
\hline
\end{tabular}

OR: odds ratio; 95\% CI: 95\% confidence interval.

0.75; 95\% CI: 0.66-0.86). Conversely, women with one or more living children (AOR: 2.07; 95\% CI: 1.59-2.70), those with $\mathrm{CD}^{+}$cell counts $\geq 351$ cells/uL (AOR: 1.25 ; 95\% CI: 1.09-1.45), and those with no prior history of tuberculosis infection (AOR: 1.17; 95\% CI: 1.01-1.35) had higher odds of dual method use.

\section{Discussion}

We successfully implemented a peer-led counseling intervention in ART clinics, focusing on dual family planning method use to prevent unintended pregnancies and STIs. Our initial projections were that 20,000 HIV-infected women 
TABLE 3: Predictors of dual method use among HIV-infected women aged 16-50 years receiving antiretroviral treatment at 16 public-sector clinics in Lusaka, Zambia (November 2009-November 2010).

\begin{tabular}{|c|c|c|c|c|}
\hline & $\begin{array}{l}\text { Women using dual } \\
\text { methods } \\
N=2,247\end{array}$ & $\begin{array}{l}\text { Women not using dual } \\
\text { methods } \\
\qquad N=9,111\end{array}$ & Crude OR $(95 \% \mathrm{CI})$ & Adjusted OR (95\% CI) \\
\hline \multicolumn{5}{|l|}{ Age at 1st visit (years) } \\
\hline $16-24$ & $170(7.6 \%)$ & $566(6.2 \%)$ & 1.00 & 1.00 \\
\hline $25-34$ & $1,269(56.5 \%)$ & $4,478(49.1 \%)$ & $0.94(0.79-1.13)$ & $0.80(0.62-1.04)$ \\
\hline$\geq 35$ & $808(36.0 \%)$ & $4,067(44.6 \%)$ & $0.66(0.55-0.80)$ & $0.51(0.41-0.63)$ \\
\hline \multicolumn{5}{|l|}{ Marital status } \\
\hline Married & $1,407(77.9 \%)$ & $5,096(71.7 \%)$ & 1.00 & 1.00 \\
\hline Single/divorced/widowed & $400(22.1 \%)$ & $2,014(28.3 \%)$ & $0.72(0.64-0.81)$ & $0.75(0.66-0.86)$ \\
\hline \multicolumn{5}{|l|}{ Education } \\
\hline None & $562(27.0 \%)$ & $2,300(27.4 \%)$ & 1.00 & 1.00 \\
\hline Primary & $692(33.2 \%)$ & $2,801(33.4 \%)$ & $1.01(0.89-1.14)$ & $1.02(0.86-1.21)$ \\
\hline Secondary or more & $830(39.8 \%)$ & $3,278(39.1 \%)$ & $1.04(0.92-1.17)$ & $1.06(0.90-1.24)$ \\
\hline \multicolumn{5}{|l|}{ Number of living children } \\
\hline 0 & $154(8.4 \%)$ & $887(12.4 \%)$ & 1.00 & 1.00 \\
\hline$\geq 1$ & $1,685(91.6 \%)$ & $6,262(87.6 \%)$ & $1.55(1.30-1.85)$ & $2.07(1.59-2.70)$ \\
\hline \multicolumn{5}{|l|}{ Monthly income } \\
\hline$<$ ZMK 200,000 & $538(35.0 \%)$ & $2,171(36.1 \%)$ & 1.00 & 1.00 \\
\hline$\geq \mathrm{ZMK} \mathrm{200,000}$ & $999(65.0 \%)$ & $3,836(63.9 \%)$ & $1.05(0.93-1.18)$ & $0.93(0.82-1.07)$ \\
\hline \multicolumn{5}{|l|}{$\mathrm{CD}^{+}$cell count (cells/uL) } \\
\hline$<250$ & $438(19.7 \%)$ & $2,035(22.5 \%)$ & 1.00 & 1.00 \\
\hline $250-350$ & $394(17.7 \%)$ & $1,648(18.2 \%)$ & $1.11(0.96-1.29)$ & $1.05(0.87-1.26)$ \\
\hline$\geq 351$ & $1,397(62.7 \%)$ & $5,349(59.2 \%)$ & $1.21(1.08-1.37)$ & $1.25(1.09-1.45)$ \\
\hline \multicolumn{5}{|l|}{ Hemoglobin (g/dL) } \\
\hline$\leq 8.0$ & $28(1.3 \%)$ & $140(1.6 \%)$ & 1.00 & 1.00 \\
\hline $8.1-9.9$ & $120(5.4 \%)$ & $590(6.5 \%)$ & $1.02(0.65-1.60)$ & $1.01(0.51-2.02)$ \\
\hline$\geq 10.0$ & $2,076(93.3 \%)$ & $8,279(91.9 \%)$ & $1.25(0.83-1.89)$ & $1.31(0.80-2.16)$ \\
\hline \multicolumn{5}{|l|}{ History or current tuberculosis } \\
\hline Yes & $333(14.8 \%)$ & $1,666(18.3 \%)$ & 1.00 & 1.00 \\
\hline No & $1,914(85.2 \%)$ & $7,445(81.7 \%)$ & $1.29(1.13-1.46)$ & $1.17(1.01-1.35)$ \\
\hline \multicolumn{5}{|c|}{ HIV status disclosure to partner } \\
\hline Yes & $1,291(57.5 \%)$ & $4,694(51.5 \%)$ & 1.00 & 1.00 \\
\hline Unknown & $956(42.5 \%)$ & $4,417(48.5 \%)$ & $0.79(0.72-0.86)$ & $1.03(0.85-1.25)$ \\
\hline \multicolumn{5}{|l|}{ Partner's HIV status } \\
\hline Negative & $102(4.5 \%)$ & $374(4.1 \%)$ & 1.00 & 1.00 \\
\hline Positive & $564(25.1 \%)$ & $2,136(23.4 \%)$ & $0.97(0.76-1.23)$ & $0.92(0.69-1.22)$ \\
\hline Unknown & $721(32.1 \%)$ & $2,636(28.9 \%)$ & $1.00(0.79-1.27)$ & $1.03(0.70-1.51)$ \\
\hline Missing & $860(38.3 \%)$ & $3,965(43.5 \%)$ & $0.80(0.63-1.00)$ & $0.89(0.65-1.22)$ \\
\hline
\end{tabular}

OR: odds ratio; $95 \%$ CI: $95 \%$ confidence interval.

would access care across the participating clinics during the 12-month implementation period. Our team of peer educators counseled 18,407 HIV-infected women enrolled in care during this period, demonstrating the feasibility of this approach in a low-resource, African setting. At their baseline counseling visit, nearly $60 \%$ of women reported use of a modern contraceptive method; the majority used solely condoms. Among contraceptive users, only $26.5 \%$ reported use of a highly effective modern contraceptive method. Dual method use was also low at $17.7 \%$.

Older, single women with lower $\mathrm{CD}^{+}$cell counts were less likely to be using modern contraception. These findings highlight a vulnerable population of HIV-infected women. As the health of women with lower $\mathrm{CD}^{+}$cell counts improves on ART and as unmarried women enter new sexual partnerships, both groups remain at risk for 
unintended pregnancy and STIs. Future reproductive health policy and programming should be expanded beyond a focus on married, healthy women [17].

We were disappointed at the low numbers of women who desired access to family planning services and were unable to obtain it in a timely fashion. We can only speculate as to the reasons for this. In Lusaka, family planning services are not integrated within HIV clinics, and most family planning clinics are only open to patients in the afternoons. A woman who has already spent much of her morning in an ART clinic is less likely to return to spend another half-day in a family planning clinic. Periodic stockouts of reproductive health commodities may also limit women's access to family planning services. Additionally, we speculate that most messages in the community promote condom use over use of more effective modern contraception or dual method use. Providers' attitudes towards sexual and reproductive healthcare for HIV-infected patients [18, 19] and misconceptions regarding the safety of hormonal contraceptive methods among women on ART may also play a role in limiting HIV-infected women's access to family planning [20].

The strengths of our study include the successful implementation of a standardized and comprehensive reproductive health counseling intervention. This intervention was acceptable to both providers and patients. Using a peer educator model, we demonstrate that it is possible to reach a sizeable number of HIV-infected women attending public ART clinics. It also became evident through our intervention that strengthening reproductive health care requires stronger linkages between family planning clinics, STI services, and HIV screening and treatment [21], as well as improved community understanding. Our study had several limitations. Contraceptive method and condom use were self-reported and thus subject to bias. Additionally, we did not assess the impact of our counseling intervention on pregnancy rates.

This study confirms the findings of smaller Zambian clinical trials $[12,22,23]$ and demonstrates that $40 \%$ of HIVinfected women on ART are not using any form of modern contraception. Among contraceptive users, less than 30\% use highly effective modern methods and even fewer use dual methods. Our counseling intervention improved uptake of modern contraceptives and dual method use modestly. Demographic and Health Surveys have demonstrated that knowledge of modern contraception is high among Zambians [24]. Additional quantitative and qualitative research should address fertility desires and barriers to dual method use in this population, guiding future public health interventions. Male partner involvement through couples counseling should be investigated further [25].

Our findings also underscore important challenges faced by HIV-infected women attempting to access family planning services within the public health system. Unmet contraceptive need is as high as $40 \%$ among these women. Strengthening public-sector service provision, with the goal of ensuring that no woman is turned away from a family planning clinic empty handed, should be an urgent priority. Finally, integrating family planning service provision into
HIV care and treatment clinics will likely improve access to reproductive health services [26].

\section{Conclusions}

We successfully demonstrate the feasibility of integrating comprehensive reproductive health counseling into HIV care and treatment. We confirm that nearly $60 \%$ of HIV-infected women receiving care within the Zambian public-sector report use of a modern contraceptive method. However, only $27 \%$ of these women use highly effective hormonal or longterm reversible methods, and even fewer women report dual method use. Additional efforts are needed to promote dual method use, particularly among older, unmarried women and those with more advanced HIV disease. These interventions should be coupled with health system changes that address critical bottlenecks in reproductive health service provision.

\section{Acknowledgments}

Programmatic funding was provided by the Tides Foundation (TFR08-02603). Additional investigator support was provided by the National Institutes of Health through the International Clinical Research Fellows Program at Vanderbilt University (R24 TW007988) and through a Clinical Scientist Development Award from the Doris Duke Charitable Foundation (2007061). Funding agencies played no role in study design, data collection, data analysis, or preparation of the paper.

\section{References}

[1] United Nations, "Millenium development goals," http://www. un.org/millenniumgoals/.

[2] W. Cates Jr., "Family planning: the essential link to achieving all eight Millennium Development Goals," Contraception, vol .81 , no. 6 , pp. $460-461,2010$.

[3] World Health Organization, Trends in Maternal Mortality: 1990 to 2008, World Health Organization, Geneva, Switzerland, 2010.

[4] Guttmacher Institute, Facts on Investing in Family Planning and Maternal and Child Heath, Guttmacher Institute, New York, NY, USA, 2010.

[5] S. Singh, J. Darroch, L. Ashford, and M. Vlassoff, Adding It Up: The Costs and Benefits of Investing in Family Planning and Maternal and Newborn Health, Guttmacher Institute, New York, NY, USA, 2009.

[6] D. Hubacher, I. Mavranezouli, and E. McGinn, "Unintended pregnancy in sub-Saharan Africa: magnitude of the problem and potential role of contraceptive implants to alleviate it," Contraception, vol. 78, no. 1, pp. 73-78, 2008.

[7] M. C. Hogan, K. J. Foreman, M. Naghavi et al., "Maternal mortality for 181 countries, 1980-2008: a systematic analysis of progress towards Millennium Development Goal 5," The Lancet, vol. 375, no. 9726, pp. 1609-1623, 2010.

[8] UNAIDS and World Health Organization, 2008 Global Report of the AIDS Epidemic, World Health Organization, Geneva, Switzerland, 2008. 
[9] Q. Abdool-Karim, C. AbouZahr, K. Dehne et al., "HIV and maternal mortality: turning the tide," The Lancet, vol. 375, no. 9730, pp. 1948-1949, 2010.

[10] World Health Organization, PMTCT Strategic Vision 2010 2012, World Health Organization, Geneva, Switzerland, 2010.

[11] S. Aboud, G. Msamanga, J. S. Read et al., "Genital tract infections among HIV-infected pregnant women in Malawi, Tanzania and Zambia," International Journal of STD and AIDS, vol. 19, no. 12, pp. 824-832, 2008.

[12] K. Grabbe, R. Stephenson, B. Vwalika et al., "Knowledge, use, and concerns about contraceptive methods among serodiscordant couples in Rwanda and Zambia," Journal of Women's Health, vol. 18, no. 9, pp. 1449-1456, 2009.

[13] R. Stephenson, K. Grabbe, B. Vwalika et al., "The influence of informed consent content on study participants' contraceptive knowledge and concerns," Studies in Family Planning, vol. 41, no. 3, pp. 217-224, 2010.

[14] Central Statistical Office, “2010 Census,” 2011, http://www .zamstats.gov.zm/.

[15] E. M. Stringer, N. T. Chintu, J. W. Levy et al., "Declining HIV prevalence among young pregnant women in Lusaka, Zambia," Bulletin of the World Health Organization, vol. 86, no. 9, pp. 697-702, 2008.

[16] J. J. Schatz, “Zambia's health-worker crisis," The Lancet, vol. 371, no. 9613, pp. 638-639, 2008.

[17] Implementing Best Practices Initiative, "Family planning for health and development: actions for change," Meeting Report, http://www.who.int/reproductivehealth/publications/family_ planning/fp_health_dvlpt/en/index.html/.

[18] S. R. Hayford and V. Agadjanian, "Providers' views concerning family planning service delivery to HIV-positive women in Mozambique," Studies in Family Planning, vol. 41, no. 4, pp. 291-300, 2010.

[19] J. Harries, D. Cooper, L. Myer, H. Bracken, V. Zweigenthal, and P. Orner, "Policy maker and health care provider perspectives on reproductive decision-making amongst HIV-infected individuals in South Africa," BMC Public Health, vol. 7, article 282, 2007.

[20] S. Adamchack, B. Janowitz, J. Liku, E. Munyambanza, T. Grey, and E. Keyes, Study of Family Planning and HIV Integrated Services in Five Countries, Family Health International, Research Triangle Park, NC, USA, 2010.

[21] R. Wilcher and W. Cates, "Reproductive choices for women with HIV," Bulletin of the World Health Organization, vol. 87, no. 11, pp. 833-839, 2009.

[22] R. Stephenson, B. Vwalika, L. Greenberg et al., "A randomized controlled trial to promote long-term contraceptive use among HIV-serodiscordant and concordant positive couples in Zambia," Journal of Women's Health, no. 4, pp. 567-574, 2011.

[23] K. E. Mark, J. Meinzen-Derr, R. Stephenson et al., "Contraception among HIV concordant and discordant couples in Zambia: a randomized controlled trial," Journal of Women's Health, vol. 16, no. 8, pp. 1200-1210, 2007.

[24] Central Statistical Office, Ministry of Health, Tropical Diseases Research Centre, University of Zambia, and Marco International, Zambia Demographic and Health Survey, CSO and Macro Interntional, Calverton, Md, USA, 2009.

[25] C. MacPhail, A. Pettifor, S. Pascoe, and H. Rees, "Predictors of dual method use for pregnancy and HIV prevention among adolescent South African women," Contraception, vol. 75, no. 5, pp. 383-389, 2007.
[26] R. Wilcher and W. Cates Jr., "Reaching the underserved: family planning for women with HIV," Studies in Family Planning, vol. 41, no. 2, pp. 125-128, 2010. 


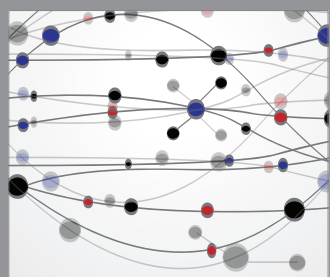

The Scientific World Journal
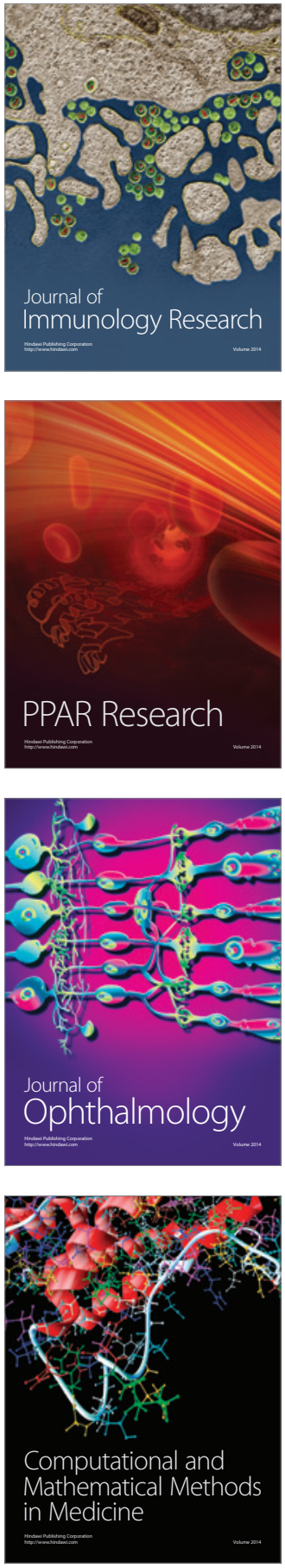

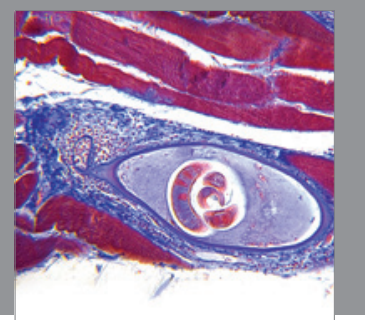

Gastroenterology

Research and Practice
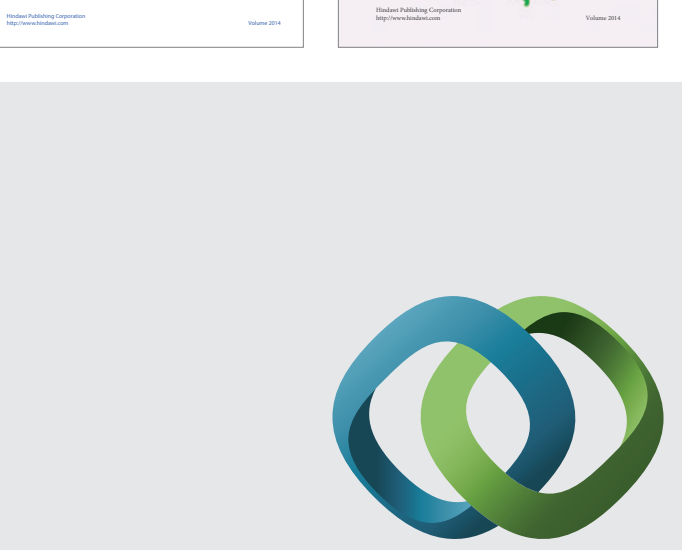

\section{Hindawi}

Submit your manuscripts at

http://www.hindawi.com
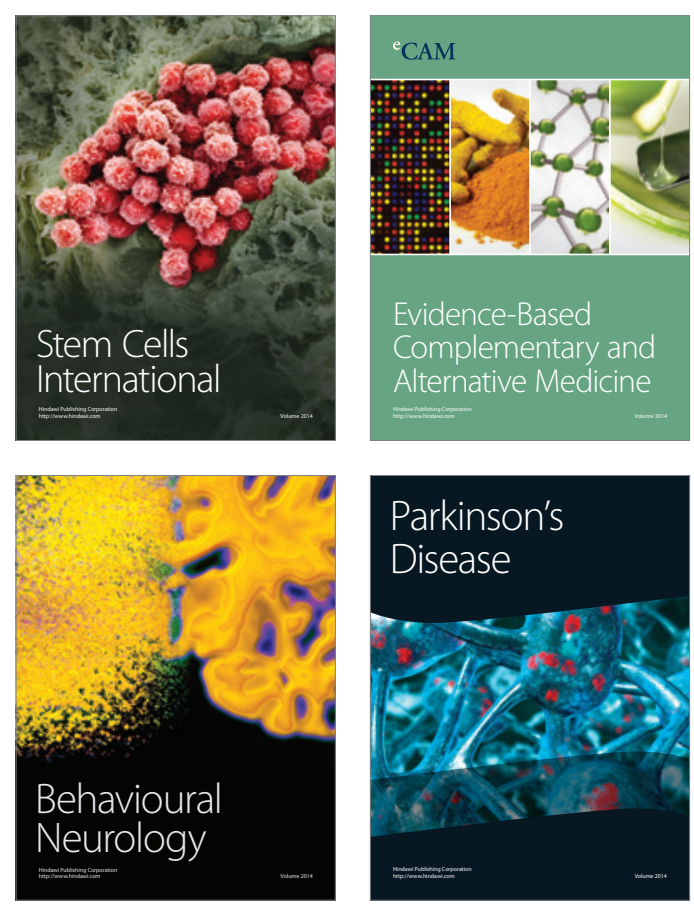

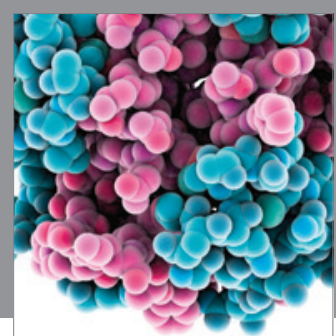

Journal of
Diabetes Research

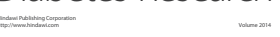

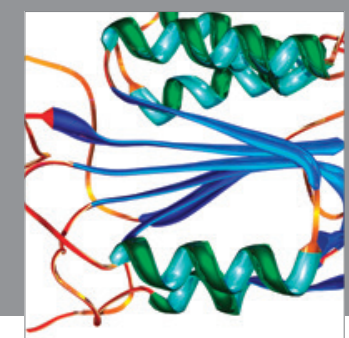

Disease Markers
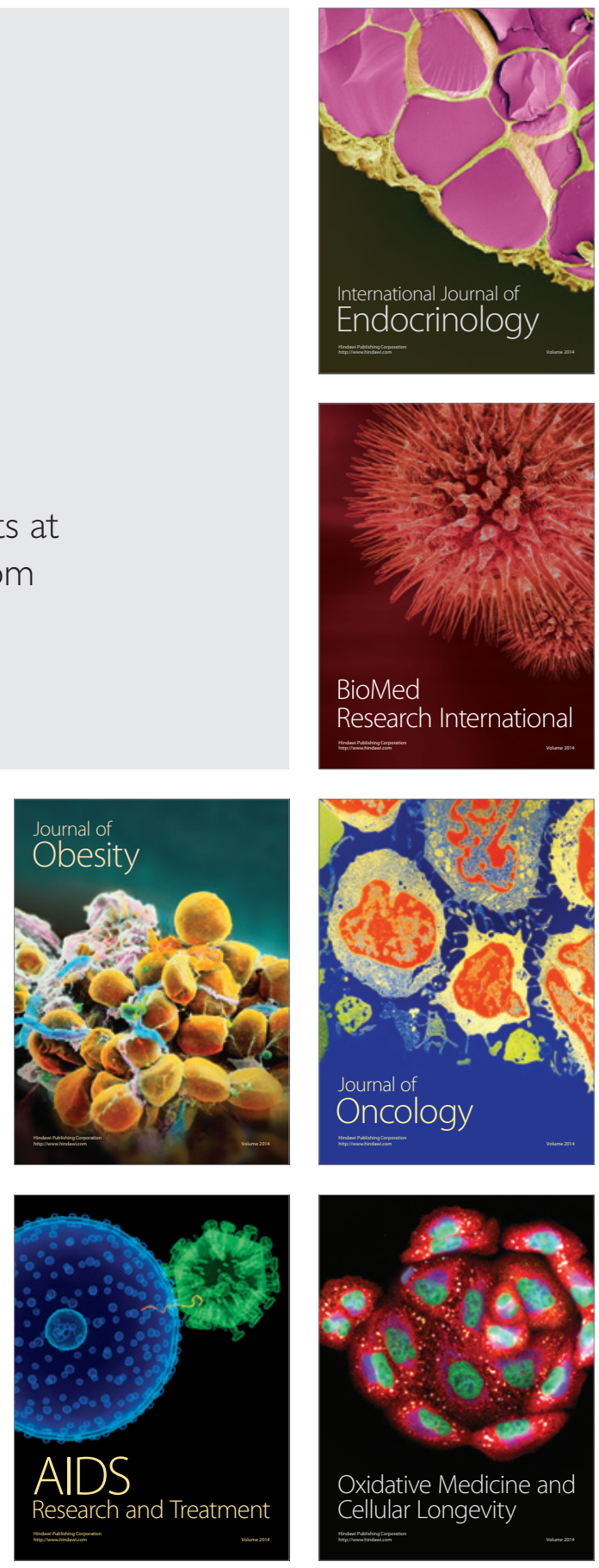